\title{
Health Hazards from High Power Electrical Lines Electromagnetic Field (EMF) Exposure in Riyadh Area Saudi Arabia
}

\author{
Mahmoud Hassan El-Bidawy ${ }^{1,2}$, Mounir Zeerban ${ }^{3}$, Waheed Mahmoud Alharizi ${ }^{4}$ \\ ${ }^{1}$ Department of BMS, Facultyof Medicine, Prince Sattam Ibn Abdulaziz University, Al Kharj, Saudi Arabia \\ ${ }^{2}$ Department of Medical Physiology, Faculty of Medicine, Cairo University, Cairo, Egypt \\ ${ }^{3}$ Department of Surgery, Faculty of Medicine, Prince Sattam Ibn Abdulaziz University, Al Kharj, SaudiArabia \\ ${ }^{4}$ Department of BMS (Biochemistry Division), Faculty of Medicine, Prince Sattam Ibn Abdulaziz University, Al kharj, Saudi Arabia
}

Email address:

melbidawy2005@hotmail.com (M. H. El-Bidawy)

To cite this article:

Mahmoud Hassan El-Bidawy, Mounir Zeerban, Waheed Mahmoud Alharizi. Health Hazards from High Power Electrical Lines

Electromagnetic Field (EMF) Exposure in Riyadh Area Saudi Arabia. American Journal of Health Research. Vol. 6, No. 4, 2018, pp. 98-102. doi: 10.11648/j.ajhr.20180604.17

Received: October 3, 2018; Accepted: October 19, 2018; Published: November 10, 2018

\begin{abstract}
In almost $90 \%$ of the cases of childhood leukemia, the etiology is unclear. A wide range of factors, including environmental, sociological, lifestyle influences are implicated as well as genetic susceptibility. The evidence is mixed as to whether (Electromagnetic Fields) EMFs can be responsible for direct DNA damage. DNA damage could arise as a result of persistently elevated free radical concentrations, caused by long-term EMF exposure. Some experiments have shown that EMFs affect these functions, though they have been difficult to reproduce and therefore remain controversial. The overall evidence is that magnetic fields induce changes in apoptosis (cell death). Changes in B lymphocytes can also change cellular division rates. A series of studies demonstrated EMF effects that made cells more likely to become cancerous. These findings may prove particularly important with regard to B-lineage (Acute Lymphocytic Leukemia) ALL. However, the original results have not yet been replicated, perhaps pointing to the need to tighten experimental protocols. In body, tissue free radicals are dangerous high-energy particles that damage cells and can both cause and accelerate the progression of cancer. Exposure to EMFs can increase the yield of free radicals by more than $60 \%$, which could explain the diverse and often inconsistent nature of observed effects of EMFs, free radicals being intermediates in many natural processes. The hormone, melatonin is thought to protect the body from cancer by (a) neutralizing free radicals, (b) inhibiting the uptake of growth factors by cancer cells, (c) by increasing the likelihood of cancer cells undergoing apoptosis (cell death), and (d) by inhibiting the growth of blood vessels in tumors. The production of melatonin at night (when the majority of melatonin is produced by the body's pineal gland) was reduced significantly by light at night and magnetic fields associated with the electricity supply. It was found that melatonin protects cells from genetic damage.
\end{abstract}

Keywords: Electro Magnetic Field, Cancer, Leukemia, High Power Electric Lines, Melatonin

\section{Introduction}

Just as certain chemical toxicants may induce expression of abnormal genes [1], recent research is exploring potential epigenetic influence of (Electromagnetic Radiation) EMR, by its impact on genetic expression [2] adverse(Electromagnetic Fields) EMFs, may serve as a trigger for the expression of pathological and disease causing genes.
Direct damage to the DNA of human lymphocytes [3] and alterations of the phagocytic activity in animal macrophages [4] has been confirmed recently and may account for changes in immunological parameters and for the immune system dysfunction attributed to EMF exposure. With alterations of cellular structures and impairment of cellular functions by these different mechanisms, it is not surprising that tissue disorders, organ dysfunction and clinical illness may ensue. Attenuation of insulin secretion characteristically found in 
diabetes, for example, can be induced or accentuated by exposure to adverse EMF through distortion of calcium influx in the cells [5]. Some investigations have explored potential EMF disturbance of blood-brain barrier with increased susceptibility to CNS toxicants [6]. Particular attention, however has recently been devoted to researching the impact of EMF on pineal gland physiology [7]. The pineal gland secretes the neuroendocrine hormone melatonin that is synthesized from the neurotransmitter serotonin. Melatonin is involved with regulation of myriad of physiological processes including sleep pattern [8], free radical metabolism [9] blood pressure control [10] nitric oxide physiology [11], lipid metabolism [12] immune system functioning [13] and activity of sex hormones as estrogen [14].

\section{Materials and Methods}

\subsection{Experimental Protocol}

(1) A sample of 150 males residents near high power electrical line in Riyadh area, Saudi Arabia. We selected one gender to avoid the gender variability of melatonin hormone secretion levels, age range between (20-70 years old) (the mean age 38years old), volunteers are living near a $725-\mathrm{kV}$ power line at distances (0-99metes, 100-199 meters ) and ( 200-299 meters ) respectively, were compared with 145 males the same age group living away from any power lines. Participants provided morning urine samples on 2 consecutive days and wore a magnetic dosimeter for 36 consecutive hours to measure personal magnetic exposure. The indoor electric field was assessed by spot measurements. After adjustment for other factors associated with low melatonin secretion, such as medication use or light exposure, nighttime concentration of 6-hydroxymelatonin was similar in the two groups. When either 24-hour or sleep-time exposure to magnetic field or electric field measurements was used, no exposure-effect relation was evident. However, the trend of decreasing 6-hydroxymelatonin concentration with age was more pronounced for males living near the lines, as was a lower 6-hydroxymelatonin concentration in males with high body mass index. Chronic residential exposure to magnetic fields from high-power electrical lines may accentuate the decrease in melatonin secretion observed in some vulnerable subgroups of the population. A written consent was taken from all participants and none invasive tests where carried out.

6-hydroxy melatonine sulfate concentrations in urine were assayed using a commercially available RIA kit (Stockgrand Ltd., Guildford, UK), which is based on a method described by Arendt and co-workers (15).

(2) Reviewing the files in local hospital near major sources of EMF for major health effects in the past year (recorded cases of leukemia).

(3) Determining the least and safest distance from high power electrical lines based on number of cases of leukemia and whether the birth residence of cases of leukemia was near or far from high power electrical lines

\subsection{Stastical Analysis}

Data are expressed as Mean \pm S. E. M. Statistical Evaluation was performed by One-Way Analysis of Variance (ANOVA), followed by Turkey Test for Multiple Comparisons. All analyses were performed with SPSS Software Package (Version 18). P $<0.05$ was selected as the Criterion for Statistical Significance

\section{Result Analysis}

The results are shown in the following table and figures.

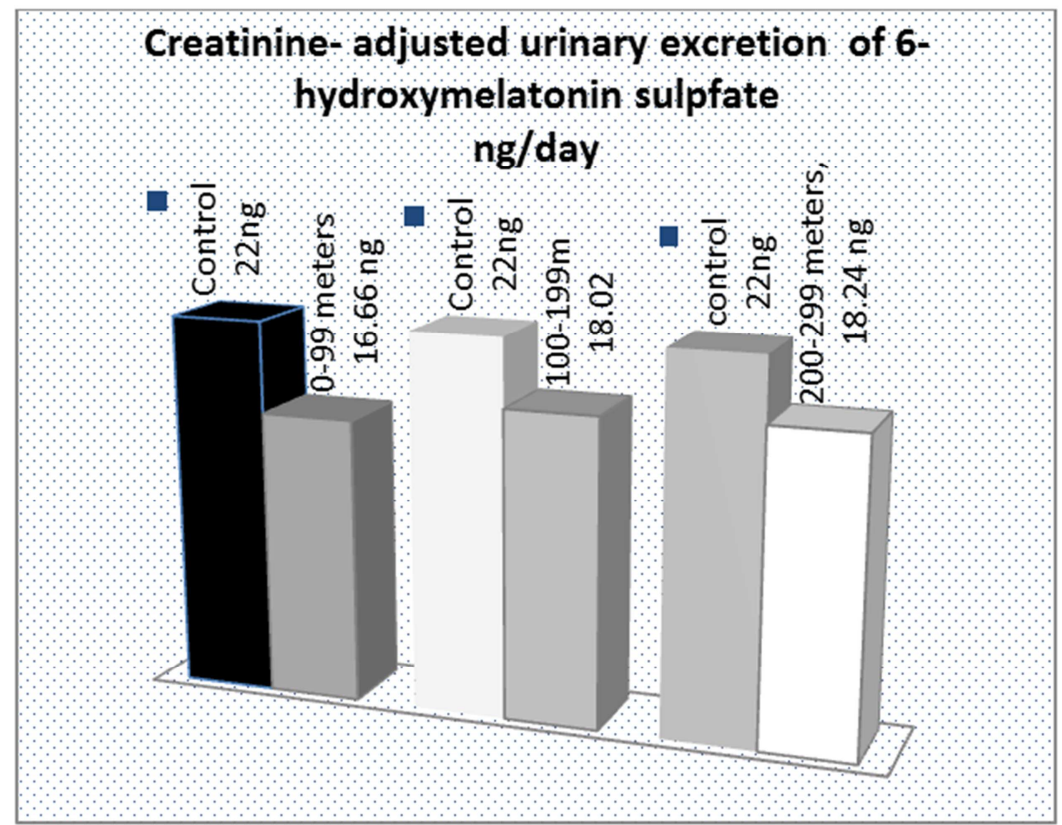

Figure 1. Effects of chronic (>1year) EMF exposure at home on the urinary excretion of Creatinine - adjusted 6-hydroxyxymelatonin sulfate concentration. 
Table 1. (Effects of chronic (>1year) EMF exposure at home on the urinary excretion of Creatinine-adjusted6-hydroxymelatonin sulfate concentration).

\begin{tabular}{lllll}
\hline Magnetic Field Exposure distance & $\begin{array}{l}\text { Control (Residents } \\
\text { away from high power } \\
\text { electrical line) }\end{array}$ & $\begin{array}{l}\text { (Residents 0-99 } \\
\text { meters from high } \\
\text { power electrical line })\end{array}$ & $\begin{array}{l}\text { (Residents 100-199 } \\
\text { meters meters from high } \\
\text { power electrical line) }\end{array}$ & $\begin{array}{l}\text { (Residents 200-299meters } \\
\text { meters from high power } \\
\text { electrical line) }\end{array}$ \\
\hline $\begin{array}{l}\text { Effect on urinary excretion o Creatinine } \\
\text {-adjusted 6- hydroxyl melatonin sulfate }\end{array}$ & $\begin{array}{l}\text { 2. ng/day } \\
(18.3-26.1) n g / \text { day }\end{array}$ & $\begin{array}{l}16.66 \\
(13.15,21.10) \mathrm{ng} / \mathrm{day}\end{array}$ & $\begin{array}{l}18.02 \\
(14.41,22.53) \mathrm{ng} / \mathrm{day}\end{array}$ & $18.24(14.53,22.89) \mathrm{ng} / \mathrm{day}$ \\
\hline
\end{tabular}

1. Birth address (less than, $<100$ meters distance from high power electric line), Urinary excretion of 6hydroxymelatonin sulfate $/ 24$ hours urine, is significantly decreased(16.66 ng) $(\mathrm{P}<0.05)$ compared to control $(22.8 \mathrm{ng})$

2. Birth address ( from 100-199 meters distance from high power electric line), Urinary excretion of 6 hydroxymelatonin sulfate $/ 24$ hours urine, is significantly decreased(18.02 ng) $(\mathrm{P}<0.05)$ compared to control $(22.8 \mathrm{ng})$

3. Birth address (equal or more than $\geq 200$ meters distance from high power electric line), Urinary excretion of 6 hydroxymelatonin sulfate/24 hours urine, is significantly decreased (18.24 ng) $(\mathrm{P}<0.05)$ compared to control (22.8 ng)

There is significant decrease in 6- hydroxyl melatonin sulfate excretion in urine $(\mathrm{P}<0.05)$ in birth residents $(0-99$ meters from high power electrical line) compared to other birth residents, the decrease in 6- hydroxyl melatonin sulfate excretion in urine is not significant between birth residents 100-199 meters and 200-299 meters from high power electrical lines)

\section{Risk of Leukaemia at different distances from nearest high power electric line}

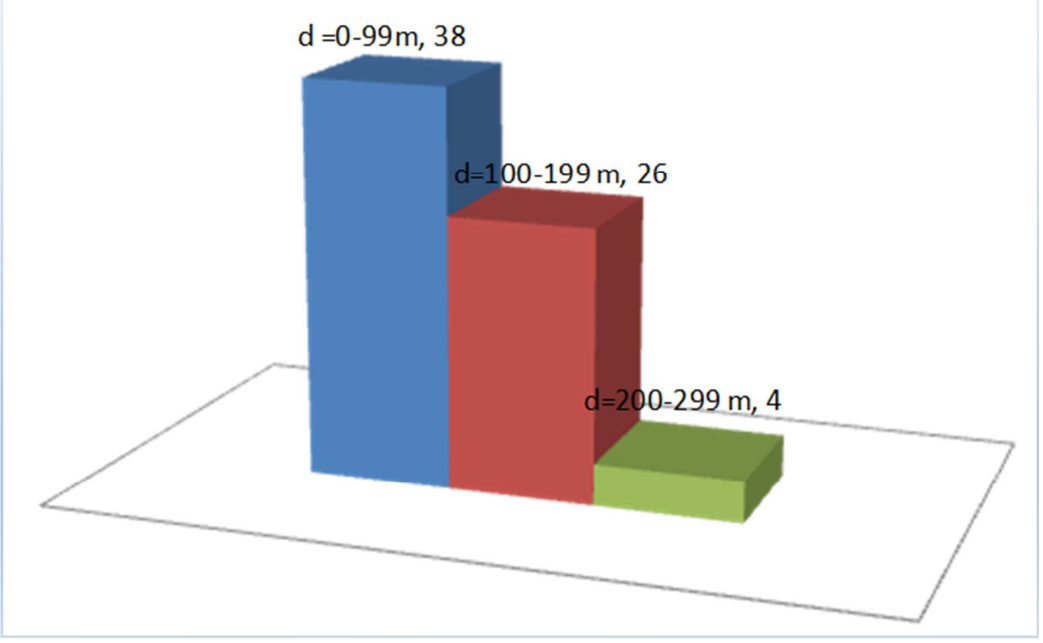

Figure 2. Distance (d) from the nearest line that the risk depends on the distance for leukaemia, the results were significant $(P<0.051)$.

Distance $(d)$ from the nearest high power electric line that the risk depends on the distance, for leukemia the number of cases increase with distance $<200$ meters, compared with higher distances $>200$ meters, the results were significant $(\mathrm{P}<$ $0.05)$.

The distribution of Leukemia cases based on their residence at birth from the nearest high power electrical lines, The relative risk is an estimate of the incidence compared with the distance, at distances, at each distance category $<200 \mathrm{~m}$ the relative risks are greater, there is some evidence that the risk varies according to distance from the line, though there is no smooth trend.

Table 2. Number of cases recorded having leukemia in the past year with records in local hospital with birth residence and there distance from nearest high power electrical lines

\begin{tabular}{ll}
\hline $\begin{array}{l}\text { Birth residence distance from high power electrical } \\
\text { line (meters)( d) }\end{array}$ & $\begin{array}{l}\text { Number of } \\
\text { Cases }\end{array}$ \\
\hline $0-99(<100$ meters $)$ & 38 \\
$100-199$ meters & 26 \\
$200-299(\geq 200$ and $<300$ meters $)$ & 4 \\
\hline
\end{tabular}

\section{Discussion}

In this work, it was found that the relative risk of leukemia was higher for children whose home address at birth was within $200 \mathrm{~m}$ of a high voltage power line compared with those more than $200 \mathrm{~m}$ from the nearest line. For 200-300 m the relative risk was relatively less. The finding that the increased leukemia risk apparently extends so far from the line is surprising in view of the very low level of magnetic field that could be produced by power lines at these distances.

There is no obvious source of bias in the choice of cases or controls. The study is based on records of childhood cancer in Riyadh. Registration for childhood cancer is related to proximity of birth address to high power electric transmission lines.

Controls were selected from registers compiled through the legally required process of birth registration. No participation by cases or controls was required except for urine analysis for end metabolite of melatonin hormone secretion in urine 
6-hydroxy melatonin sulfate

Populations near power lines may have different characteristics from the rest of the population. In control data there is a slight tendency in urban areas for greater affluence closer to lines, though in rural areas there is no clear trend. There is known to be a positive association between affluence and rates of childhood leukemia. However, adjustment for socioeconomic status of the census ward of birth address did not explain these findings. Population mixing has been associated with childhood leukemia, [8] but in studied cases, individual mobility, measured by changes of postcode between birth and diagnosis, was no more common for those whose home at birth was closer to the lines. Other characteristics of the population (for instance parity, which has sometimes been found to be associated with childhood leukemia [9] may vary with proximity to power lines.

The results are highly significant but could nevertheless be due to chance-for example, if the leukemia controls are not sufficiently representative of the relevant population. Some support for this explanation can be derived from the different distance distributions observed for the leukemia and non-leukemia residents

Studies included in the pooled analysis referred to above contain, or have been extended to include, analysis of proximity to power lines [10]. Of these, one, a previous UK study, with 1582 cases of leukemia diagnosed during 1992, in this study it was found that a relative risk of 1.42 ( 0.85 to 2.37$)$ for acute lymphocytic leukemia within $400 \mathrm{~m}$ for 275 and 400 $\mathrm{kV}$ lines; this supports the current results. Studies in Canada [11] and Sweden [7] also found increased risks for childhood leukemia (Canada: relative risk 1.8 (0.7 to 4.7) for residence within $100 \mathrm{~m}$ of transmission lines of $50 \mathrm{kV}$ or more, and 1.3 within $50 \mathrm{~m}$; Sweden: 2.9 (1.0 to 7.3 ) for residence $50 \mathrm{~m}$ versus 101-300 m from 220 and $400 \mathrm{kV}$ power lines, with no increase for other childhood cancers). Studies from Denmark, [12] Norway, [13] and the United States [14] found relative risks below 1.0 but were based on smaller numbers. The current study estimates also relates to individuals residents distance from high power electric lines, some used a reference category that is within the distance where current study found an increased risk.

This study concerned home address at birth, whereas much previous magnetic field epidemiology has concerned address at other times. Half of the children with leukemia in this study had the same address at diagnosis as at birth; but no corresponding information for the control group.

The most obvious explanation of the association with distance from a line is that it is indeed a consequence of exposure to magnetic fields. The increased risk seemed to extend to at least $200 \mathrm{~m}$, and at that distance typical calculated fields from power lines are $<0.1 \mu \mathrm{T}$, and often $<0.01$ $\mu \mathrm{T}$ - that is, less than the average fields in homes from other sources.

The significant decrease in the melatonin hormone excretory product 6- hydroxymelatonin in urine, in males living near high power electrical lines, and this decreased secretion is proportionate with the decrease in distance may indicated a link between the higher risk of developing malignancies in these persons more than the other population as the potential link between disordered melatonin physiology and the development of malignancy has emerged as a priority area of investigation particularly in breast and prostate cancer, melanoma, colon cancer, lung cancer and leukemia [15]. Also a variety of bone marrow cells have been shown to produce melatonin. Whilst the specific function of melatonin in these cells remains unknown, its suppression could have clear implications for leukemia initiation and / or progression. A reduction in melatonin in the leukocyte precursor cells would be expected to enhance free radical-mediated DNA damage.

\section{Conclusion}

An association between leukemia and power lines has been reported in several studies, but it is nevertheless surprising to find the effect extending so far from the lines. There is no satisfactory explanation for the results in terms of causation by magnetic fields or association with other factors. No previous findings relating to level of exposure to magnetic fields are supported by convincing laboratory data or any accepted biological mechanism. The potential link between disordered melatonin physiology, with decreased its excretory metabolic end product in urine (6 hydroxymelatonin sulfate) and the development of malignancy has emerged as a priority area of investigation particularly in breast and prostate cancer, melanoma, colon cancer, lung cancer and leukemia. Assuming that the higher risk in the vicinity of high voltage lines is indeed a consequence of proximity to the lines we can estimate the attributable annual number of cases of leukemia in Riyadh area. Power frequency magnetic fields, produced by the high power electric system, are "possibly carcinogenic".

\section{Acknowledgements}

This project was supported by the deanship of scientific research at Prince Sattam Ibn Abdulaziz University, AlKharj, KSA. under the research project number (2014/03/2181).

\section{References}

[1] Crew D, McLachlan JA. Epigenetics, evolution, endocrine disruption, health, and disease, Endocrinology 006:147(Suppl):S4-S10.

[2] Blank M, Goodman R. Intial interaction in electromagnetic field-induced biosynthesis. J CellPhysiol 2004:199:359-63.

[3] Delimaris J, Tsilimigaki S, Messini-Nicolaki N, Ziros E, Piperakis SM. Effects of pulsed electricfield on DNA of human lymphocytes. Cell BiolToxicol 2006;22:40-15.

[4] Frahm J, Lantow M, Lupke M, Weiss DG, Smiko M. Alteration in cellular functins in mousemacrophages after exposure to $50 \mathrm{~Hz}$ magnetic fields. J Cell Biochem 2006;99:168-77. 
[5] Sakurai T, Satake A, Sumi S, Inoue, Miyakoshi J. An extremely low frequency manetic fieldattenuates insulin secretion from the insulinoma cell line, RI-m. Bioelectromagnetics2004;25:160-6.

[6] Cherry N. Potential and actual adverse effects of radiofrequency and microwave radiation atlevels near and below 2 microW/cm2. Lincoln:New Zealand Lincoln University;199. Available at:Cherry Environmental Health Consulting website <http://www.neilcherry.com/cart/> [accessed8 January 2007].

[7] Raindra T, Lakshmi NK, Ahuja YR. Melatonin in pathogenesis and therapy of cancer. Indian JMed Sci 2006;60:523-35.

[8] Pandi-Peumal SR, Srinivasan V, Maestroni GJ, Cardinal DP, Poeggeler B, Hardeland R. Melatonin:nature's most versatile biological signal? FEBS J 2006;273:2813-38.

[9] Poeggeler B, Saarela S, Reiter RJ, et al. Melatonin -a highly poentendogenou radicalscavenger and electron donor: new aspects of the oxidation chemistry of this indole accessedin vitro. Ann N Y acadSci 1994;738:419-20.

[10] Cagnacci A, Cannoletta M, Renzi A, Baldassari F, Arangino S, Volpe A. Prolomged melatoninadministration decreases nocturnal blood pressure in women. Am J Hypert 2005;18:1614-8.
[11] Pozo D, Reiter RJ, Calvo JR, Guerero JM. Physiological concentrations of melatonin inhibitnitric oxide synthase in rat cerebellum. Life Sci 1994;55:PL455-60.

[12] Cherry N. Criticism in health assessment in the ICNIRP guidlines for radiofrequency andmicrowave radiation (100 kHz-300GHz). Lincoln:New Zealand University;2002. Available at:Cherry Environmental Health Consulting Website $<$,http://www.neilcherry.com/cart/Major+evidence+Review?m ode=show_category $>$ [accessed 10 January 2007].

[13] Walleczek J. Electromagnetic field effects on cells of the immune system:the role of calciumsignaling. Faseb J $1992 ; 6: 3177-85$.

[14] Adriaens I, Jacquet P, Cortvrindt R, Janssen K, Smitz. Melatonin has dose-dependent effectson folliculogenesis, oocyte maturation capacity and steroidogenesis. Toxicology 2006;228:333-43.

[15] BojkowskiCJ, ArendtJ. 1990Factors influencing urinary6-sulphatoxymelatonin, a major melatonin metabolite, in normal human subjects. Clin Endocrinol (Oxf).33:435-444. 